\title{
Biometrics, JABES and the International Biometric Society
}

\author{
Elizabeth THOMPSON, Marie DAVIDIAN, and Stephen BUCKLAND
}

The Biometric Society was founded 70 years ago, officially formed on September 6, 1947, with a mission to promote the development and application of statistical and mathematical theory and methods in the biosciences, including agriculture, biomedical science and public health, ecology, environmental sciences, forestry, and allied disciplines. In 70 years, the Society has come a long way. Now, the International Biometric Society (IBS) is a worldwide society with almost 6000 members from over 70 different countries. The Society contains 35 recognized Regions, and four inter-regional Networks. Over half of IBS Regions represent members from developing countries.

\footnotetext{
Biometry:

the active pursuit of biological knowledge by quantitative methods

(Fisher, 1948)

2017 Statement of the Society:

The International Biometric Society cherishes the diversity of our membership, who represent over 70 different countries, with a wide range of ethnic, cultural and religious heritages. Any differences are transcended through our shared interest in statistical and mathematical methods for the biosciences.

Our different perspectives challenge each other and bring greater depth to our work. Intellectual exchange within our society is fostered by the meetings that are routinely held by the various regions that make up our Society, as well as our biennial International Biometric Conference. The Society places the highest priority on our members' freedom to attend such meetings and to engage with each other on a global scale.
}

As we celebrate the 70th birthday of the IBS and its ethnic, gender, geographic, and disciplinary diversity, we celebrate also the two scientific journals of the Society: Biometrics and $\boldsymbol{J A B E S}$, which are the outward face of the Society and support the IBS mission to promote quantitative biosciences education and research capacity building worldwide.

Elizabeth Thompson Seattle, USA (E-mail: eathomp@u.washington.edu). Marie Davidian Raleigh, USA (E-mail: marie_davidian@ncsu.edu). Stephen Buckland (凶) St Andrews, UK (E-mail: steve@st-andrews.ac.uk). 
Table 1. Regional origins of new journal submissions in 2016.

\begin{tabular}{lcc}
\hline \hline & Biometrics & JABES \\
\hline Total 2016 new submissions & 617 & 161 \\
Percentage of submissions & & \\
Africa & 0.5 & 11.8 \\
Asia & 15.3 & 47.2 \\
Europe & 17.4 & 13.7 \\
Latin America and Caribbean & 3.1 & 6.2 \\
North America & 61.2 & 13.8 \\
Oceania & 2.2 & 4.3 \\
\hline
\end{tabular}

Biometrics: The journal is a top scholarly publication reporting work promoting the use of statistical and mathematical theory and methods in the biosciences. Biometrics was founded in 1945 as Biometrics Bulletin, and the streamlined title Biometrics has been in use since 1947. The journal has published many seminal, highly-cited articles on a host of topics in biometry authored by the most respected authorities in the field, and is available in over 5100 institutional libraries worldwide. Reflecting the volume of submissions, Biometrics now has an executive editor, three co-editors and over 90 associate editors whose expertise spans a diverse range of specialties. Biometrics articles are typically centered on a substantive application providing an illustrative context for new methodology, and most articles are accompanied by computer code implementing the methods.

JABES: In the early 1990s, some members of IBS argued for a new journal in which standard papers were structured around a motivating example, and for which there was balance across the agricultural, biological and environmental sciences, following a steady rise in the representation of human genetics, health, and medicine in Biometrics papers. Discussions between IBS and the American Statistical Association (ASA) resulted in the launch in 1996 of JABES, jointly published by the two societies. ASA continues to be actively involved through the journal's Editorial Management Committee, although IBS is now responsible for all material aspects of the journal.

JABES has been successful at maintaining balance across the disciplines it serves, as well as a good geographic spread of authors, and thus fulfils a valuable role in helping IBS maintain a high profile internationally and across a range of disciplines that utilize biometric methodologies (see Table 1). In line with many other journals, authors are now strongly encouraged to provide computer code and data online, to ease the uptake of methods by the user community.

Every 2 years, there is an International Biometrics Conference (IBC), the major meeting of the IBS. IBCs are widely recognized as among the most diverse, scientific, and friendly, international statistical meetings, with worldwide participants at all career stages. At an IBC, member publications in Biometrics and JABES are recognized in Showcase Sessions. Each journal conducts a competition for the "Best Paper published by an IBS member", and each Showcase Session features presentations by an author of the winning papers. 
Thank you to readers of Biometrics and JABES who are IBS members. We encourage our other readers to join IBS through their Region, and we welcome all to the next IBC in Barcelona, in July 2018.

Elizabeth Thompson, IBS President

Marie Davidian, Executive Editor, Biometrics

Stephen Buckland, Editor, JABES

[Published Online August 2017.] 